\title{
Artificial Intelligence Algorithm in Mobile Robots
}

\author{
Abdusamad Al-Marghirani, \\ Northern Border University KSA
}

\begin{abstract}
The most important contemporary problems in artificial intelligence problems robots phones and mobile, to its heavy reliance on the general structure and the different movements so as to select the item clearly so that there is a great potential of to make sure the data and it is full control on the movement of the robot to give him orders and the possibility of Android implemented the concept of whatever environment changed [1]

Robots animated special, which can be moved remotely by a mobile phone to be able to carry luggage from the warehouses, which are designed in with programmable Lego that will help us in the integration of communications and allow easily use multiple sets at one time so as to allow us this programming from the use of a robot and one linked to a different set of $\mathrm{t}$ scattered and ease of handling in with artificial intelligence and communications devices to control phones and find out all the movements by using intelligent software orders [2.3]
\end{abstract}

\section{Key Words}

Color Learning, Robot Vision.. Mobile robots, algorithms

\section{INTRODUCTION}

In devices Acrobat moving in with mobile Android based move from one area to enter the exit area easily using artificial intelligence and smart software for high quality level, especially when the robot move and sort the desired and upload it to the area's most accurate and special aircraft and warships minute purposes and then uses colors to know on luggage to be transferred blue, red and yellow in order to sort them is before the transfer and delivery to the intended target [4]

The following figure shows the entry of Android smart phone from the first to the log out after identifying colors that are needed to sort the data so that it can reach the goal of continuously and reliably and without acquiring any missprogrammed path.

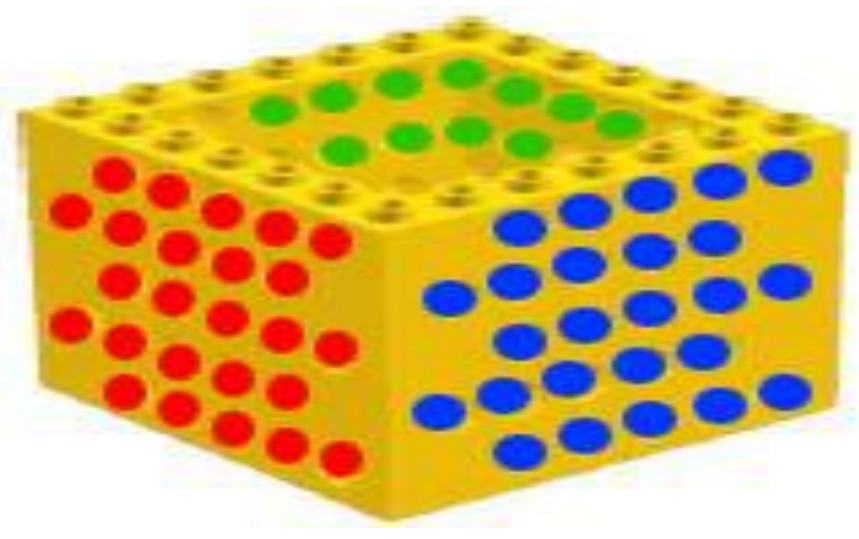

Figure 1 shows the general appearance of the exterior colors to identify the robot to sort the data and send it to the desired target.
In this figure you must know and focus on the conditions that must be available for the development of algorithms and reliable so as to select the tracks in the sort so that the system of complete control and precision big to steer track by Android mobile, sell so we get a high quality level, especially in the movement areas dangerous chemical.[5]

We also Figure 2 order items by accredited colors red, yellow and blue to determine the path from the transmitter to the desired goal and to reach out more effectively

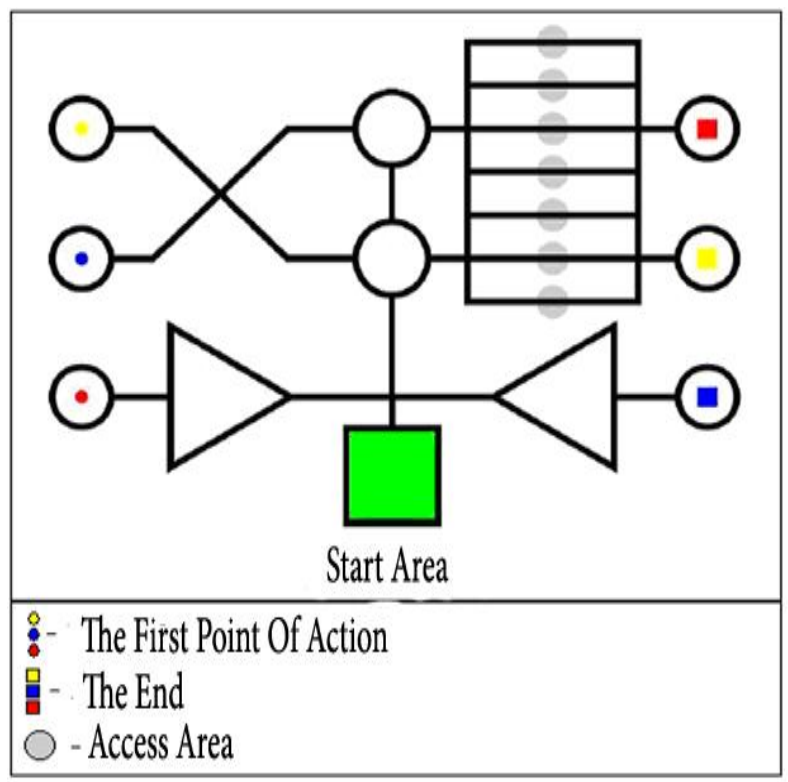

Figure 2 order items by accredited colors

\section{TYPES OF MOBILE ROBOTS}

There are different types of mobile robots, which depends on the application and the speed and type of environment, whether in space or in the water and also the terrain has been identified four main types of robotics.

\subsection{Terrestrial or ground contact robots}

It is considered the most common among the robots because they contain wheels and some other parties to be .

\subsection{Aquatic robots}

These robots are working in the water or under the water surface because it uses water fans .

\subsection{Airborne robots}

These robots that fly like helicopters, as well as fixed-wing aircraft or balloons or umbrellas that control automatically by.

\subsection{Space robots}

These robots have been designed for outer space so attractive to have a small and usually are for the maintenance of the international space station and space robots move by climbing or be independent.

\section{PROBLEM STATEMENT}

Mobile robots problem, especially modern ones lies in. 
1. Identifying objects and operations that must be carried out in order to be able to identify them properly and without errors

2. The problem of sorting special attention should be paid to ensure the sustainability of the formations in the presence of dynamic errors, resources. Ensure the development of a reliable algorithm MR behavior when driving on-line, object detection and counting

3. Key specifications for the control and reliability and large capacity to learn and interact with the changing environment of the problems to make sure that dynamic as possible to produce errors in determining the tracks

4. The development of a reliable algorithm behavior

\section{APPROCH}

In this study was to solve problems posed by designing a high-level of Mobile Robots LEGO and it uses scripts with high specifications Bricks

Was illustrated in Figure 3 android mobile in one easy without any symptoms and to determine the colors and do in learning and reading and identifying tracks without any undergone a movement[6]

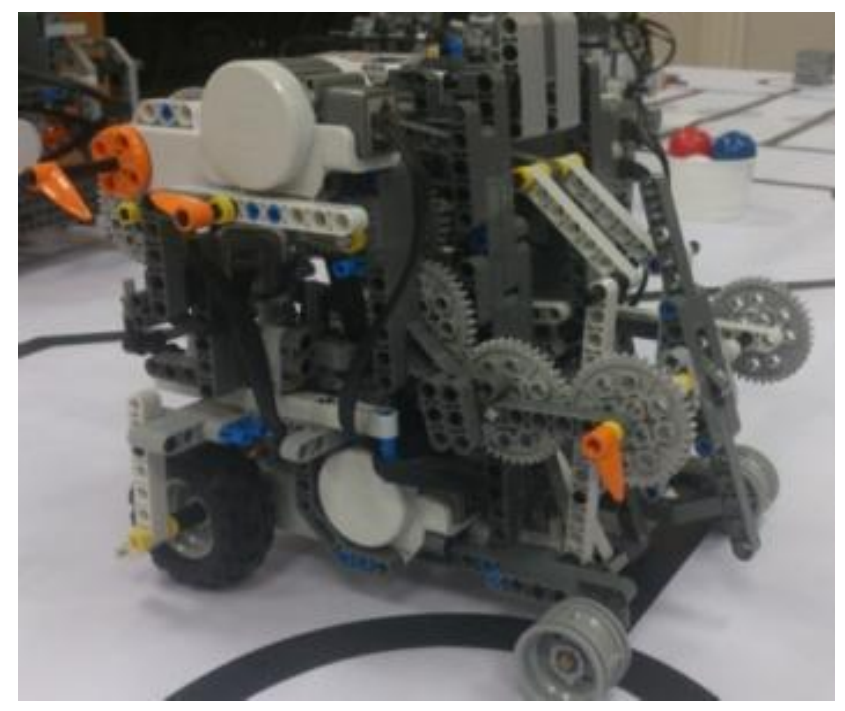

Figure 3 High-level of Mobile Robots LEGO

Definition scripts and selects the tracks

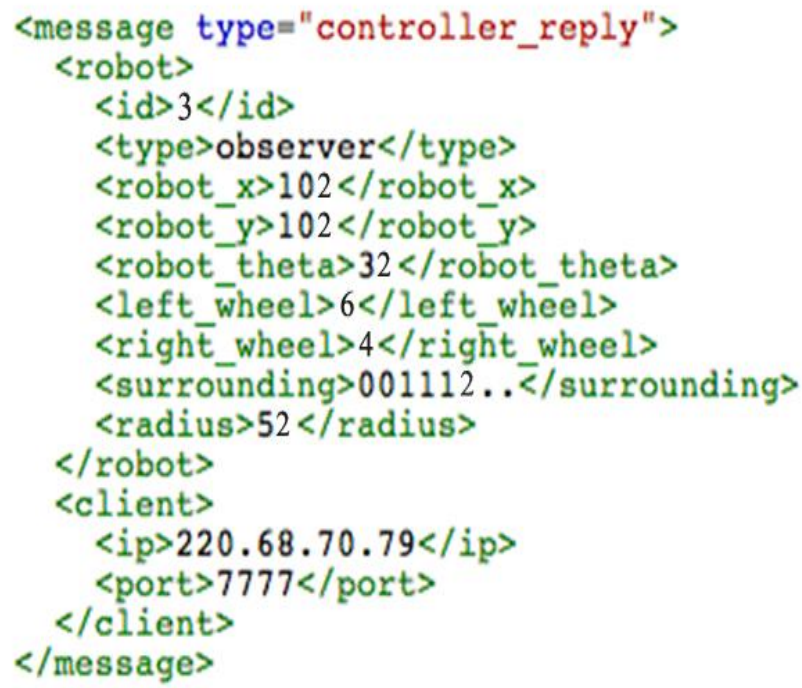

Ways representation of the colors in the Mobile Robots In order to identify the robots of the required elements need to a set of points colors $(! 3[0 ; N ; 2])$. Complete mapping edentates a color label for each point

in the color space:

(2) $8 p ; q ; r 2[0 ; 255]$;

$f C 1 ; p ; C 2 ; q ; C 3 ; r g$ 7! !j!2[0;N;2]

where $C 1 ; C 2 ; C 3$ are the color channels

(e.g. RGB, Cyber), with the corresponding

Values ranging from $0 ; 255$

A complete mapping edentates a color label for each point in the color space:

(2 8p; q; r 2 [0; 255];

$f C 1 ; p ; C 2 ; q ; C 3 ; r g$ ?! !j!2[0;N;1].

Where $C 1 ; C 2$; $C 3$ are the color channels (e.g. RGB, $\mathrm{YCbCr}$ ), with the corresponding

Values ranging from $0 ; 255$.

\section{GENERAL TRACK USING ARTIFICIAL INTELLIGENCE}
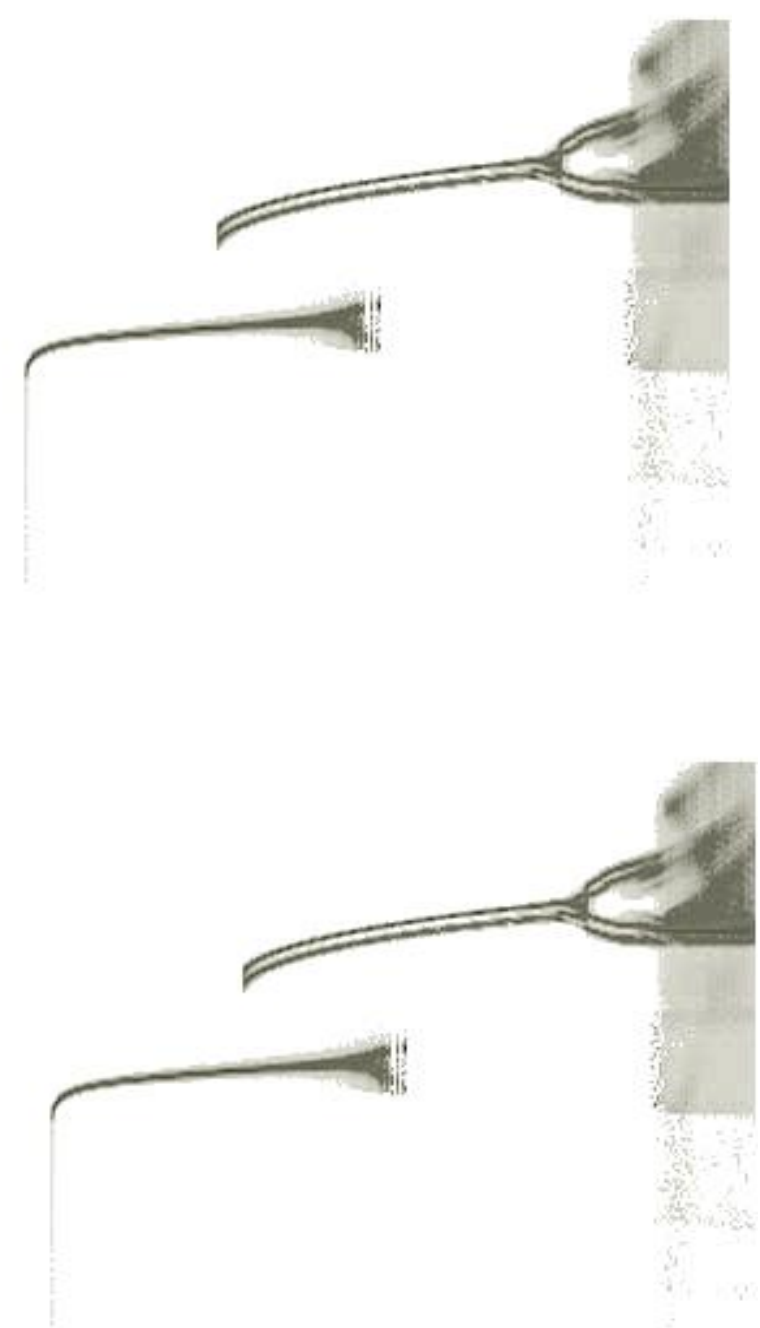
Artificial intelligence have a very large impact on the work of the robot is based on a central long time ago, but after manufacturing remotely became very important computer because prices and cost become more low, especially in the storage, sorting, and especially from the point the beginning to the end so that work becomes harmonic is large, effective, and get great quality and by high.

Mobile robots is one of the advanced technologies developed that help humans in the purposes and tasks in many different areas such as education, services, industry, transport and much more in the field of observation, exploration and use robots to explore the environment of what is new and is not reachable by humans and the best example of this ROBOVOLC is one of the robots, which are designed to explore and measurement in volcanic environment because it may be dangerous for the volcanoes in the application and industry, and is used mainly mobile robot to transport materials from one point to another. Is known as the Automated Guided Vehicle (AGV). Using AGV in the industry, and can reduce the cost and efficiency can be increased. Depends on the mobile robot sensors to obtain information about the environment around them usually, has been equipped mobile robot with various types of sensors in order to guide the robot to achieve its purpose. Some devices, sensors, and image devices, or a combination of both remote sensing and capture devices to capture the image can be treated as a hardware support in the navigation of robots. These data image capture devices from these devices and the use of vision algorithms to analyze the terrain (Howard et al, 2001) or sensor fusion to translate sensory input in various reliable estimates and environmental models .

While most research robots with the naked eye to see, touch, and there are also some problems with logic and adapt and respond to the environment of the many variables that have been solved with the help of artificial intelligence techniques using heuristic such as ANN.

Have been proposed neural computers to provide a higher level of intelligence that allows for such robots to work in the natural environment as well as to perform tasks in a natural environment. The concept of control when the robots are a well-established fields of discipline which intelligent control system to include independent interactions with the environment. The definition of intelligence as the ability to act appropriately in an unstable environment where the appropriate action in this environment is that increases the probability of success in these conditions and survival, here is a sub-goals that support the ultimate goal of the system and act in intelligent systems so as to achieve the maximum degree of Possibility is the establishment of goals and standards that indicate success in the external environment of the system smart phone, which are capable of sensing the environment, which can be achieved through the use of sensors and then visualize and explain the situation in order to make decisions through careful analysis and implementation of well control procedures appropriate by the use of motor drives solid, which is where the process of storage and require levels higher than the intelligence capabilities to identify the objects and store events and the use of knowledge about the world and plan the future and learn why and quality
, and there are also forms of advanced intelligence, which have the ability to understand, analyze, and planning and choice wisely and also planning successfully in a competitive environment and society hostile .

Here you will consider that intelligent behavior is a crucial matter for most of the mobile robots can support this idea from conception through to work

\section{CONCLUSIONS}

In this study after confirmation of the results show that the mobile robot has a great capacity to resolve the multiple problems and deal with them easily without getting any 1 quality and dramatically as he shows great potential to control and focus on the points Distributed various areas.

Of course there are a lot of problems which depend on labor and employment, especially direct action and determine the required targets without causing any problems Of the hardest parts in any scripts that rely on parts of the algorithm, especially in the smart devices that apply to mobile and mobile robot, especially in determining the color of the first episode definitional transfer tracks the main task of which is associated with tracks from Sub-controlled system .

\section{REFERENCES}

[1] [Asterisk : The Definitive Guide, 4th Edition] Russell Bryant, Leif Madsen, Jim Van Meggelen, O'Reilly, May 2013, ISBN :978-1-4493-3242-6

[2] [Switching to VoIP] Theodore Wallingford, O'Reilly, June 2005, ISBN10 :0-596-00868-6

[3] Intelligent Robots and Systems (IROS), 2012 IEEE/RSJ International Conference on

[4] Ay, N., Bertschinger, N., Der, R., G"uttler, F., and Olbrich, E. (2008). Predictive information and explorative behavior of autonomous robots. The European Physical Journal B, 63(3):329-339

[5] Janzing, D. and Balduzzi, D. (2012). Quantifying causal influences. arXiv preprint arXiv:1203.6502,

[6] M. Jungel. Using layered color precision for a selfcalibrating vision system. In The Eighth International RoboCup Symposium, Lisbon, Portugal, 2004

[7] Shuvra Das, Sandra A. Yost, Mohan Krishnan, "A 10Year Mechatronics Curriculum Development Initiative: Relevance, Content and Results-Part I", IEEE Transactions on Education, vol.53, no.2, pp.194-201, May. 2010.

[8] Gómez-de-Gabriel, J.M., Mandow, A., FernándezLozano, J., García- Cerezo, A.J. "Using LEGO NXT Mobile Robots With LabVIEW forUndergraduate Courses on Mechatronics", IEEE Transactions o Education, vol.54, no.1, pp.41-47, Feb. 2011.

[9] N. Agmon and D. Peleg. Fault-tolerant Gathering Algorithms for Autonomous Mobile Robots. In Proc. of the 15th ACM-SIAM Symposium on Discrete Algorithms, pages $1070-1078,2004$. 